Young Shin Kim, Jaesung Lee, Stefan Mittnik, and Jiho Park

\title{
Quanto Option Pricing in the Presence of Fat Tails and Asymmetric Dependence
}

Working Paper Number 1 1, 2013

Center for Quantitative Risk Analysis (CEQURA)

Department of Statistics

University of Munich

http://www.cequra.uni-muenchen.de 


\title{
Quanto Option Pricing in the Presence of Fat Tails and Asymmetric Dependence
}

\author{
Young Shin Kim*, Jaesung Leeł Stefan Mittnik; Jiho Park ${ }^{\S}$
}

November 12, 2013

\begin{abstract}
We present an approach to pricing European quanto options assuming that the underlying instruments follow a multivariate normal tempered stable (NTS) process. This allows both fat-tailedness and asymmetric dependence between the returns on the underlying asset and the exchange rate to be accommodated. In an empirical application, we estimate the market and risk-neutral parameters for a quanto construction involving the Nikkei 225 index, as the underlying asset, and the Japanese yen and U.S. dollar exchange rate. While the BlackScholes model is clearly rejected by the data, the NTS model cannot be rejected at any reasonable level. Deriving quanto call and put prices under both NTS and Black-Scholes assumptions from the estimated models, we find that the resulting prices differ substantially, with the NTS model yielding generally higher prices as it better reflects the tail properties of the instruments involved.
\end{abstract}

Keywords: multivariate normal tempered stable process, Lévy process, Black-Scholes option pricing, Nikkei 225 dollar options

${ }^{*}$ College of Business, Stony Brook University, New York, USA

${ }^{\dagger}$ Department of Mathematics, Sogang University, Korea

${ }^{\ddagger}$ Corresponding author: Center for Quantitative Risk Analysis, Department of Statistics, Ludwig Maximilians University Munich, Akademiestr. 1/I, 80799 Munich, Germany, finmetrics@stat.uni-muenchen.de

$\S_{\text {Department of Mathematics, Sogang University, Korea }}$ 


\section{Introduction}

A standard quanto option is a foreign-currency European option, whose payoff at maturity is converted to another (typically domestic) currency at a fixed exchange rate. The main purpose of using quantos is to provide foreignasset exposure without taking the corresponding exchange rate risk. As a consequence, the dependence structure between the asset and the exchange rate is instrumental in the valuation of quantos. Quanto pricing based on the Black-Scholes model (Black and Scholes, 1973), assuming a multivariate Brownian motion as data-generating process, has been studied, among others, by Baxter and Rennie (1996) and Dimitroff et al. (2009). Recently, Park et al. (2013) proposed a method for pricing quanto options in the stochastic volatility setting considered in Hull and White (1987), using the series expansion approach introduced in Antonelli et al. (2010). Apart from allowing for stochastic volatility, their setup remains in the classical Black-Scholes setting. The Black-Scholes assumptions have, however, been soundly rejected on empirical grounds due to the fat-tailedness and the asymmetry commonly observed in returns on financial assets (cf. Rachev and Mittnik (2000), Morales (2007) and Harmantzis and Miao (2009)).

In this paper, we present an approach to pricing quanto options under the assumptions that the financial instruments involved are governed by a multivariate normal tempered stable (NTS) process. NTS distributions and processes have been introduced by Barndorff-Nielsen and Levendorskii (2001), using a time-changed Brownian motion with a tempered stable subordinator, and applied in Barndorff-Nielsen and Shephard (2001). The multivariate NTS distribution has been studied empirically and applied to portfolio analysis in Kim et al. (2012). The asymmetric dependence of the NTS distribution 
is discussed in Kim and Volkmann (2013).

Given that the NTS model is expected to provide a better description of the empirically observed return processes by permitting fat-tailedness and asymmetric dependence, it is also expected to give rise to more realistic option valuation. Pricing European and American call and put options for Lévy processes, a general class containing the NTS process, has been extensively discussed in the literatures, including Carr and Madan (1999), Lewis (2001) Barndorff-Nielsen and Levendorskii (2001), and Kim et al. (2008). Studies on quanto options seem to be lacking, however. In order to conduct quanto option pricing, two-dimensional processes need to be considered. Given their stronger empirical footing, multivariate NTS processes appear to provide a suitable framework for quanto option valuation.

In the following, we present a closed-form solution for quanto option pricing under the NTS model. An empirical application, involving the Nikkei 225 index and the exchange rate between the U.S. dollar and the Japanese yen, illustrates that the NTS model clearly dominates the Black-Scholes model, which, in contrast to the NTS model, is soundly rejected by the data. We find that the quanto call and put prices derived under NTS assumptions can substantially differ from those derived in a Black-Scholes setting. The latter tends to underestimate the underlying risk and, thus, leads to lower quanto prices.

The remainder of this paper is organized as follows. In Section 2, we discuss the multivariate NTS distribution and the change of measures for the NTS process. Section 3 reviews quanto option pricing in the BlackScholes framework, before presenting the solution for the NTS model. An illustrative empirical application is given in Section 4. We summarize our 
main findings in Section 5.

\section{Multivariate NTS Processes}

We consider an $N$-dimensional process and define $\alpha \in(0,2), \theta>0, \gamma=$ $\left(\gamma_{1}, \gamma_{2}, \ldots, \gamma_{N}\right)^{\top} \in \mathbb{R}^{N}, \beta=\left(\beta_{1}, \beta_{2}, \ldots, \beta_{N}\right)^{\top} \in \mathbb{R}^{N}$, and $\sigma=\left(\sigma_{1}, \sigma_{2}, \ldots, \sigma_{N}\right)^{\top}$,

with $\sigma_{n}>0$, for all $n \in\{1,2, \ldots, N\}$. Let $R=\left[\rho_{m, n}\right]_{m, n \in\{1,2, \ldots, N\}}$ be a dispersion matrix and $R^{1 / 2}$ given by factorization $R=R^{1 / 2}\left(R^{1 / 2}\right)^{\top}$, such as a Cholesky factorization, and $\mathcal{T}=(\mathcal{T}(t))_{t \geq 0}$ be a tempered stable subordinator with characteristic function

$$
\phi_{\mathcal{T}(t)}(u)=\exp \left(-\frac{2 t \theta^{1-\frac{\alpha}{2}}}{\alpha}\left((\theta-i u)^{\frac{\alpha}{2}}-\theta^{\frac{\alpha}{2}}\right)\right)
$$

and let $B=(B(t))_{t \geq 0}$ be an independent $N$-dimensional Brownian motion, i.e., $B(t)=\left(B_{1}(t), B_{2}(t), \ldots, B_{N}(t)\right)^{\top}$, which is assumed to be independent of $\mathcal{T}$.

The $N$-dimensional process $X=(X(t))_{t \geq 0}, X(t)=\left(X_{1}(t), X_{2}(t), \ldots, X_{N}(t)\right)^{\top}$, defined by

$$
X(t)=\gamma t+\beta(\mathcal{T}(t)-t)+\operatorname{diag}(\sigma) R^{1 / 2} B(\mathcal{T}(t))
$$

is called an $N$-dimensional NTS process and denoted by

$$
X \sim \operatorname{NTS}_{N}(\alpha, \theta, \gamma, \beta, \sigma, R)
$$

The characteristic function of $X_{n}$ is

$$
\phi_{X_{n}(t)}(u)=\exp \left(\left(\gamma_{n}-\beta_{n}\right) i u t-\frac{2 t \theta^{1-\frac{\alpha}{2}}}{\alpha}\left(\left(\theta-\beta_{n} i u+\frac{\sigma_{n}^{2} u^{2}}{2}\right)^{\frac{\alpha}{2}}-\theta^{\frac{\alpha}{2}}\right)\right) ;
$$

and expectation and covariance are given by

$$
E\left[X_{n}(t)\right]=\gamma_{n} t
$$


and

$$
\operatorname{cov}\left(X_{m}(t), X_{n}(t)\right)=\sigma_{m} \sigma_{n} \rho_{m, n} t+\beta_{m} \beta_{n} t\left(\frac{2-\alpha}{2 \theta}\right),
$$

respectively. The characteristic function is analytically extended in a subset of the complex field

$I_{n}=\left\{z \in \mathbb{C}:-\frac{1}{\sigma_{n}^{2}}\left(\beta_{n}+\sqrt{\beta_{n}^{2}+2 \sigma_{n}^{2} \theta}\right) \leq \operatorname{Im}(z) \leq \frac{1}{\sigma_{n}^{2}}\left(\sqrt{\beta_{n}^{2}+2 \sigma_{n}^{2} \theta}-\beta_{n}\right)\right\}$,

that is, $\phi_{X_{n}(t)}(\xi)$ is well defined, if $\xi \in I_{n}$.

As the following proposition states, ${ }^{1}$ weighted sums of the NTS random variables are again NTS distributed.

Proposition 2.1. Let $w=\left(w_{1}, w_{2}, \ldots, w_{N}\right)^{\top} \in \mathbb{R}^{N}$ and $X \sim \operatorname{NTS}_{N}(\alpha, \theta, \gamma, \beta$, $\sigma, R)$. Then, $w^{\top} X \sim \operatorname{NTS}_{1}(\alpha, \theta, \bar{\gamma}, \bar{\beta}, \bar{\sigma}, 1)$ with

$$
\bar{\gamma}=\sum_{n=1}^{N} w_{n} \gamma_{n}, \quad \bar{\beta}=\sum_{n=1}^{N} w_{n} \beta_{n}, \quad \text { and } \quad \bar{\sigma}=\sqrt{\sum_{m=1}^{N} \sum_{n=1}^{N} w_{m} w_{n} \sigma_{m} \sigma_{n} \rho_{m, n} .}
$$

To discuss the change of measure for the NTS process, we recall Girsanov's theorem.

Theorem 2.1 (Girsanov, cf. Klebaner (2005)). Suppose $(B(t))_{t \geq 0}, B(t)=$ $\left(B_{1}(t), B_{2}(t), \cdots, B_{N}(t)\right)^{\top}$, is an $N$-dimensional Brownian motion under measure $\mathbb{P}$. Let $(W(t))_{t \geq 0}, W(t)=\left(W_{1}(t), W_{2}(t), \cdots, W_{N}(t)\right)^{\top}$, be an $N$-dimensional process, such that

$$
W_{n}(t)=B_{n}(t)+\int_{0}^{t} H_{n}(s) d s
$$

with $(H(t))_{t \geq 0}, H(t)=\left(H_{1}(t), H_{2}(t), \cdots, H_{N}(t)\right)^{\top}$, being an adapted process satisfying $\int_{0}^{t}\|H(s)\|^{2} d s<\infty$. Moreover, let

$$
\Xi(t)=-\sum_{n=1}^{N} \int_{0}^{t} H_{n}(s) d B_{n}(s)
$$

\footnotetext{
${ }^{1}$ See Kim et al. (2012) for the proof.
} 
and assume that $\left(e^{\Xi(t)-\frac{1}{2}[\Xi, \Xi](t)}\right)_{t \in[0, \mathcal{T}]}$ is a martingale. Then, there is an equivalent probability measure $\mathbb{Q}$, such that $(W(t))_{t \geq 0}$ is a $\mathbb{Q}$-Brownian motion. $\mathbb{Q}$ is determined by

$$
\frac{d \mathbb{Q}}{d \mathbb{P}}=e^{\Xi(T)-\frac{1}{2}[\Xi, \Xi](T)},
$$

where $[\Xi, \Xi](t)=\sum_{n=1}^{N} \int_{0}^{t}\left(H_{n}(s)\right)^{2} d s, t \in[0, T]$.

Let $(\tau(t))_{t \geq 0}$ be the process satisfying $\mathcal{T}(t)=\int_{0}^{t} \tau(u) d u$, for all $t \geq 0$. Then,

$$
X(t)=\gamma t+\beta \int_{0}^{t}(\tau(u)-1) d u+\operatorname{diag}(\gamma) R^{1 / 2} \int_{0}^{t} \sqrt{\tau(u)} d B(u)
$$

Moreover, let $\lambda=\left(\lambda_{1}, \lambda_{2}, \cdots, \lambda_{N}\right)^{\top}$ and $\hat{\beta}=\left(\hat{\beta}_{1}, \hat{\beta}_{2}, \cdots, \hat{\beta}_{N}\right)^{\top}$ be vectors satisfying

$$
\gamma-\beta=\lambda-\hat{\beta}
$$

and let $H(t)=\left(H_{1}(t), H_{2}(t), \ldots, H_{N}(t)\right)^{\top}$ be an $N$-dimensional process, such that

$$
\operatorname{diag}(\gamma) R^{1 / 2} H(t)=(\beta-\hat{\beta}) \sqrt{\tau(t)}
$$

Then,

$$
\begin{aligned}
X(t)= & \lambda t+\hat{\beta} \int_{0}^{t}(\tau(u)-1) d u \\
& +\operatorname{diag}(\gamma) R^{1 / 2}\left(\int_{0}^{t} \sqrt{\tau(u)} H(u) d u+\int_{0}^{t} \sqrt{\tau(u)} d B(u)\right) .
\end{aligned}
$$

By Theorem 2.1, with

$$
\frac{d \mathbb{Q}}{d \mathbb{P}}=e^{\Xi(T)-\frac{1}{2}[\Xi, \Xi](T)}, \quad \text { for } \quad \Xi(t)=-\sum_{n=1}^{N} \int_{0}^{t} H_{n}(s) d B_{n}(s),
$$

process

$$
W(t)=B(t)+\int_{0}^{t} H(u) d u
$$


is a $\mathbb{Q}$-Brownian motion; and we have

$$
\begin{aligned}
X(t) & =\lambda t+\hat{\beta} \int_{0}^{t}(\tau(u)-1) d u+\operatorname{diag}(\gamma) R^{1 / 2} \int_{0}^{t} \sqrt{\tau(u)} d W(u) \\
& =\lambda t+\hat{\beta}(\mathcal{T}(t)-t)+\operatorname{diag}(\gamma) R^{1 / 2} W(\mathcal{T}(t))
\end{aligned}
$$

Therefore, as the following proposition states, $X \sim \operatorname{NTS}_{N}(\alpha, \theta, \lambda, \hat{\beta}, \sigma, R)$ is an NTS-process under measure $\mathbb{Q}$.

Proposition 2.2. Suppose $X \sim \operatorname{NTS}_{N}(\alpha, \theta, \gamma, \beta, \sigma, R)$ under measure $\mathbb{P}$. Let $\lambda=\left(\lambda_{1}, \lambda_{2}, \cdots, \lambda_{N}\right)^{\top}$ and $\hat{\beta}=\left(\hat{\beta}_{1}, \hat{\beta}_{2}, \cdots, \hat{\beta}_{N}\right)^{\top}$ be vectors satisfying

$$
\gamma-\beta=\lambda-\hat{\beta}
$$

Then, there is an equivalent measure $\mathbb{Q}$, such that

$$
X \sim \operatorname{NTS}_{N}(\alpha, \theta, \lambda, \hat{\beta}, \sigma, R)
$$

In this case, the Radon-Nikodym derivative is given by (3).

Below, we will derive the quanto option price assuming that the asset and the exchange rate follow a two-dimensional NTS process.

\section{Quanto Option Pricing}

We denote the domestic and the foreign risk-free interest rates by $r_{d}$ and $r_{f}$, respectively. Then, let $(S(t))_{t \geq 0}$ be the price process for the asset in foreign currency, $(V(t))_{t \geq 0}$ the price process of the asset in domestic currency, and $(F(t))_{t \geq 0}$ the exchange rate process of the foreign currency relative to the domestic currency.

Before presenting the solution for quanto option pricing in the NTS framework, we summarize the results under Black-Scholes assumptions (cf. Section 4.5 in Baxter and Rennie, 1996). 


\subsection{Black-Scholes Quanto Option Pricing}

Suppose that the processes $V(t)$ and $F(t)$ are such that

$$
V(t)=V(0) \exp \left(\mu_{X} t+\sigma_{X} W_{X}(t)\right)
$$

and

$$
F(t)=F(0) \exp \left(\mu_{Y} t+\sigma_{Y} W_{Y}(t)\right)
$$

where $\left(W_{X}(t)\right)_{t \geq 0}$ and $\left(W_{Y}(t)\right)_{t \geq 0}$ are Brownian motions with correlation $\rho$. To simplify the model, we assume that $W_{Y}(t)=\rho W_{X}(t)+\bar{\rho} \bar{W}_{Y}(t), \bar{\rho}=$ $\sqrt{1-\rho^{2}}$ and that $W_{X}(t)$ and $\bar{W}_{Y}(t)$ are independent.

To derive a risk-neutral measure, $\mathbb{Q}$, which makes $\exp \left(-r_{d} t\right) V(t)$ and $\exp \left(-\left(r_{d}-r_{f}\right) t\right) F(t)$ martingales, we apply Girsanov's theorem and specify the market prices of risk as

$$
\lambda_{1}=\frac{1}{\sigma_{X}}\left(\mu_{X}+\frac{1}{2} \sigma_{X}^{2}-r_{d}\right)
$$

and

$$
\lambda_{2}=\frac{\rho}{\sigma_{X} \bar{\rho}}\left(-\mu_{X}-\frac{1}{2} \sigma_{X}^{2}+r_{d}\right)+\frac{1}{\sigma_{Y} \bar{\rho}}\left(\mu_{Y}+\frac{1}{2} \sigma_{Y}^{2}-r_{d}+r_{f}\right)
$$

and set $\tilde{W}_{X}(t)$ and $\tilde{\bar{W}}_{Y}(t)$ to

$$
\tilde{W}_{X}(t)=\lambda_{1} t+W_{X}(t
$$

and

$$
\tilde{\bar{W}}_{Y}(t)=\lambda_{2} t+\bar{W}_{Y}(t)
$$

respectively. Then, there is an equivalent measure $\mathbb{Q}$, such that $\left(\tilde{W}_{X}(t)\right)_{t \geq 0}$ and $\left(\tilde{\bar{W}}_{Y}(t)\right)_{t \geq 0}$ are independent Brownian motions under $\mathbb{Q}$. Hence, under measure $\mathbb{Q}$, we have

$$
V(t)=V(0) \exp \left(r_{d} t-\frac{\sigma_{X}^{2}}{2} t+\sigma_{X} \tilde{W}_{X}(t)\right)
$$


and

$$
F(t)=F(0) \exp \left(\left(r_{d}-r_{f}\right) t-\frac{1}{2} \sigma_{Y}^{2} t+\sigma_{Y} \rho \tilde{W}_{X}(t)+\sigma_{Y} \bar{\rho} \tilde{W}_{Y}(t)\right)
$$

Moreover, since $S(t)=V(t) / F(t)$, we have

$$
S(t)=S(0) \exp \left(r_{f} t-\frac{1}{2}\left(\sigma_{X}^{2}-\sigma_{Y}^{2}\right) t+\left(\sigma_{X}-\sigma_{Y} \rho\right) \tilde{W}_{X}(t)-\sigma_{Y} \bar{\rho} \tilde{\bar{W}}_{Y}(t)\right) .
$$

Let $\sigma^{2}=\sigma_{X}^{2}-2 \sigma_{X} \sigma_{Y} \rho+\sigma_{Y}^{2}$, and let $(W(t))_{t \geq 0}$ be a Brownian motion independent to $\left(\tilde{W}_{X}(t)\right)_{t \geq 0}$ and $\left(\tilde{\bar{W}}_{Y}(t)\right)_{t \geq 0}$ under measure $\mathbb{Q}$. Then, the process $\left(\left(\sigma_{X}-\sigma_{Y} \rho\right) \tilde{W}_{X}(t)-\sigma_{Y} \bar{\rho} \tilde{\bar{W}}_{Y}(t)\right)_{t \geq 0}$ is equivalent to the process $(\sigma W(t))_{t \geq 0}$ in $L^{2}$, so that

$$
\begin{aligned}
S(t) & =S(0) \exp \left(r_{f} t-\frac{1}{2}\left(\sigma_{X}^{2}-\sigma_{Y}^{2}\right) t+\sigma W(t)\right) \\
& =S(0) \exp \left(r_{f} t+\left(\sigma_{Y}^{2}-\sigma_{X} \sigma_{Y} \rho\right) t-\frac{\sigma^{2}}{2} t+\sigma W(t)\right)
\end{aligned}
$$

The payoff function of a quanto call option is given by $F_{f i x}(S(T)-K)^{+}$, where $F_{f i x}$ is the pre-determined exchange rate. Applying the Black-Scholes option pricing formula, we can compute the price of a quanto call option, $c_{0}$, at time $t=0$, via

$$
\begin{aligned}
c_{0} & =e^{-r_{d} T} E_{\mathbb{Q}}\left[F_{f i x}(S(T)-K)^{+}\right] \\
& =F_{f i x}\left(e^{\left(r_{f}-r_{d}+\sigma_{Y}^{2}-\rho \sigma_{X} \sigma_{Y}\right) T} S(0) N\left(d_{1}\right)-e^{-r_{d} T} K N\left(d_{2}\right)\right),
\end{aligned}
$$

with

$$
\begin{aligned}
& d_{1}=\frac{\left(r_{f}+\sigma_{Y}^{2}-\rho \sigma_{X} \sigma_{Y}+\frac{1}{2} \sigma^{2}\right) T+\log (S(0) / K)}{\sigma \sqrt{T}} \\
& d_{2}=d_{1}-\sigma \sqrt{T},
\end{aligned}
$$

and $N(\cdot)$ representing the cumulative standard normal distribution function. 


\subsection{NTS Quanto Option Pricing}

To obtain the NTS-based quanto option value, we assume that processes $(V(t))_{t \geq 0}$ and $(F(t))_{t \geq 0}$ are given by

$$
V(t)=V(0) \exp \left(\mu_{X} t+X(t)\right) \text { and } F(t)=F(0) \exp \left(\mu_{Y} t+Y(t)\right),
$$

where $\mu_{X}, \mu_{Y} \in \mathbb{R}$ and

$$
(X, Y) \sim \mathrm{NTS}_{2}\left(\alpha, \theta,\left[\begin{array}{l}
0 \\
0
\end{array}\right],\left[\begin{array}{c}
\beta_{X} \\
\beta_{Y}
\end{array}\right],\left[\begin{array}{l}
\sigma_{X} \\
\sigma_{Y}
\end{array}\right],\left[\begin{array}{ll}
1 & \rho \\
\rho & 1
\end{array}\right]\right)
$$

under the physical (or market) measure $\mathbb{P}$. Using Proposition 2.2, we can find equivalent measure $\mathbb{Q}_{\lambda}$, under which

$$
(X, Y) \sim \mathrm{NTS}_{2}\left(\alpha, \theta,\left[\begin{array}{c}
\lambda_{X} \\
\lambda_{Y}
\end{array}\right],\left[\begin{array}{c}
\beta_{X}+\lambda_{X} \\
\beta_{Y}+\lambda_{Y}
\end{array}\right],\left[\begin{array}{c}
\sigma_{X} \\
\sigma_{Y}
\end{array}\right],\left[\begin{array}{cc}
1 & \rho \\
\rho & 1
\end{array}\right]\right) .
$$

To derive the risk-neutral measure, we have to find an equivalent measure, $\mathbb{Q}_{\lambda^{*}}$, with $\lambda^{*}=\left(\lambda_{X}^{*}, \lambda_{Y}^{*}\right)^{\top}$, under which the discounted price processes $(\tilde{V}(t))_{t \geq 0}$ and $(\tilde{F}(t))_{t \geq 0}$, with $\tilde{V}(t)=e^{-r_{d} t} V(t)$ and $\tilde{F}(t)=e^{\left(-r_{d}+r_{f}\right) t} F(t)$, are martingales. The martingale property is satisfied, if $E_{\mathbb{Q}_{\lambda^{*}}}[\tilde{V}(t)]=V(0)$ and $E_{\mathbb{Q}_{\lambda^{*}}}[\tilde{F}(t)]=F(0)$, which is equivalent to

$$
e^{\left(\mu_{X}-r_{d}\right) t} E_{\mathbb{Q}_{\lambda^{*}}}\left[e^{X(t)}\right]=1 \text {, and } e^{\left(\mu_{Y}-r_{d}+r_{f}\right) t} E_{\mathbb{Q}_{\lambda^{*}}}\left[e^{Y(t)}\right]=1 .
$$

Hence, $\lambda^{*}$ has to satisfy:

RN.1: $\lambda_{X}^{*}<\theta-\beta_{X}-\frac{\sigma_{X}^{2}}{2}$ and $\lambda_{Y}^{*}<\theta-\beta_{Y}-\frac{\sigma_{Y}^{2}}{2}$ for $E_{\mathbb{Q}_{\lambda^{*}}}\left[e^{X(t)}\right]$ and $E_{\mathbb{Q}_{\lambda^{*}}}\left[e^{Y(t)}\right]$ to exist.

RN.2: $\mu_{X}-r_{d}+w\left(\lambda_{X}^{*}\right)=0$ and $\mu_{Y}-r_{d}+r_{f}+w\left(\lambda_{Y}^{*}\right)=0$, where

$$
\begin{aligned}
w\left(\lambda_{X}^{*}\right) & =\log E_{\mathbb{Q}_{\lambda^{*}}}\left[e^{X(1)}\right] \\
& =-\beta_{X}-\frac{2 \theta^{1-\frac{\alpha}{2}}}{\alpha}\left(\left(\theta-\beta_{X}-\lambda_{X}^{*}-\frac{\sigma_{X}^{2}}{2}\right)^{\frac{\alpha}{2}}-\theta^{\frac{\alpha}{2}}\right)
\end{aligned}
$$


and

$$
\begin{aligned}
w\left(\lambda_{Y}^{*}\right) & =\log E_{\mathbb{Q}_{\lambda^{*}}}\left[e^{Y(1)}\right] \\
& =-\beta_{Y}-\frac{2 \theta^{1-\frac{\alpha}{2}}}{\alpha}\left(\left(\theta-\beta_{Y}-\lambda_{Y}^{*}-\frac{\sigma_{Y}^{2}}{2}\right)^{\frac{\alpha}{2}}-\theta^{\frac{\alpha}{2}}\right) .
\end{aligned}
$$

Moreover, we have $\mu_{X}=r_{d}-w\left(\lambda_{X}^{*}\right)$ and $\mu_{Y}=r_{d}-r_{f}-w\left(\lambda_{Y}^{*}\right)$, so that

$$
V(t)=V(0) \exp \left(\left(r_{d}-w\left(\lambda_{X}^{*}\right)\right) t+X(t)\right)
$$

and

$$
F(t)=F(0) \exp \left(\left(r_{d}-r_{f}-w\left(\lambda_{Y}^{*}\right)\right) t+Y(t)\right) .
$$

Since the asset price in foreign currency, $S(t)$, is obtained by $S(t)=V(t) / F(t)$, we have

$$
S(t)=S(0) \exp \left(\left(r_{f}-w\left(\lambda_{X}^{*}\right)+w\left(\lambda_{Y}^{*}\right)\right) t+Z(t)\right)
$$

with $Z(t)=X(t)-Y(t)$, under the risk-neutral measure $\mathbb{Q}_{\lambda^{*}}$. By Proposition 2.1, process $Z=(Z(t))_{t \geq 0}$ follows $Z \sim \operatorname{NTS}_{1}\left(\alpha, \theta, \gamma_{Z}, \beta_{Z}, \sigma_{Z}, 0\right)$ under the measure $\mathbb{Q}_{\lambda^{*}}$, where $\gamma_{Z}=\lambda_{X}^{*}-\lambda_{Y}^{*}, \beta_{Z}=\beta_{X}+\lambda_{X}^{*}-\beta_{Y}-\lambda_{Y}^{*}$ and $\sigma_{Z}=$ $\sqrt{\sigma_{X}^{2}+\sigma_{Y}^{2}-2 \sigma_{X} \sigma_{Y} \rho}$. Hence, the characteristic function of $Z(t)$ is given by

$$
\begin{aligned}
& \phi_{Z(t)}(u)=\exp \left(-\left(\beta_{X}-\beta_{Y}\right) i u t-\frac{2 t \theta^{1-\frac{\alpha}{2}}}{\alpha} \times\right. \\
& \left.\quad\left(\left(\theta-i\left(\beta_{X}+\lambda_{X}^{*}-\beta_{Y}-\lambda_{Y}^{*}\right) u+\frac{u^{2}}{2}\left(\sigma_{X}^{2}+\sigma_{Y}^{2}-2 \sigma_{X} \sigma_{Y} \rho\right)\right)^{\frac{\alpha}{2}}-\theta^{\frac{\alpha}{2}}\right)\right) .
\end{aligned}
$$

We can now find the quanto option pricing formula using the general European option pricing formula given in Lewis (2001), giving rise to the following theorem.

Theorem 3.1. Let $h(x)$ be a payoff function of a European option with $x=\log S(T)$ and $\hat{h}(\xi)=\int_{-\infty}^{\infty} e^{-i \xi x} h(x) d x$. Suppose $\hat{h}(\xi)$ is defined for all $\xi \in$ 
$R_{h}=\left\{z \in \mathbb{C}: \operatorname{Im}(z) \in I_{h}\right\}$, for some open interval $I_{h}$. The driving process $(U(T))_{t \geq 0}$, with $U(t)=\ln S(t)$, is a Lévy process, such that a characteristic function $\phi_{U(T-t)}(u)$ of $U(T-t)$ is defined for all $\xi \in R_{\phi}=\{z \in \mathbb{C}: \operatorname{Im}(z) \in$ $\left.I_{\phi}\right\}$, for some open interval $I_{\phi}$. Then, the European option price $C(t)$ is determined by

$$
C(t)=\frac{e^{-r_{d}(T-t)}}{2 \pi} \int_{-\infty}^{\infty}(S(t))^{i(u+i \zeta)} \phi_{U(T-t)}(u+i \zeta) \hat{h}(u+i \zeta) d u
$$

where $\zeta \in I_{h} \cap I_{\phi}$.

Since the payoff function of the quanto option is given by $F_{f i x}(S(T)-K)^{+}$, we have

$$
h(x)=F_{f i x}\left(e^{x}-K\right)^{+}
$$

and

$$
\hat{h}(\xi)=-\frac{F_{f i x} K^{1-i \xi}}{\xi(\xi+i)} .
$$

Moreover, $\hat{h}(\xi)$ is well defined for $\xi \in\left\{z \in \mathbb{C}: \operatorname{Im}(z) \in I_{h}=(-\infty,-1)\right\}$. By (6) and (7), we have

$$
U(T-t)=\left(r_{f}-w\left(\lambda_{X}^{*}\right)+w\left(\lambda_{Y}^{*}\right)\right)(T-t)+Z(T-t)
$$

and, hence,

$$
\phi_{U(T-t)}(\xi)=e^{i \xi\left(r_{f}-w\left(\lambda_{X}^{*}\right)+w\left(\lambda_{Y}^{*}\right)\right)(T-t)} \phi_{Z(T-t)}(\xi)
$$

Functions $\phi_{U(T-t)}(\xi)$ and $\phi_{Z(T-t)}(\xi)$ are both well defined for $\xi \in\{z \in \mathbb{C}$ : $\left.\operatorname{Im}(z) \in I_{\phi_{Z}}\right\}$, with

$$
I_{\phi_{Z}}=\left[-\frac{1}{\sigma_{Z}^{2}}\left(\beta_{Z}+\sqrt{\beta_{Z}^{2}+2 \sigma_{Z}^{2} \theta}\right), \frac{1}{\sigma_{Z}^{2}}\left(\sqrt{\beta_{Z}^{2}+2 \sigma_{Z}^{2} \theta}-\beta_{Z}\right)\right] .
$$


Therefore, using Theorem 3.1, the European call price is determined by

$$
\begin{aligned}
C(t)= & \frac{e^{-r_{d}(T-t)}}{2 \pi} \int_{-\infty}^{\infty}(S(t))^{i(u+i \zeta)} e^{i \xi\left(r_{f}-w\left(\lambda_{X}^{*}\right)+w\left(\lambda_{Y}^{*}\right)\right)(T-t)} \\
& \times \phi_{Z(T-t)}(\xi) \frac{F_{f i x} K^{1-i(u+i \zeta)}}{(-1)(u+i \zeta)(u+i(\zeta+1))} d u \\
= & \frac{e^{-r_{d}(T-t)} F_{f i x} K^{1+\zeta}}{2 \pi S(t)^{\zeta} e^{\zeta\left(r_{f}-w\left(\lambda_{X}^{*}\right)+w\left(\lambda_{Y}^{*}\right)\right)(T-t)}} \\
& \times \int_{-\infty}^{\infty} \frac{S(t)^{i u} e^{i u\left(r_{f}-w\left(\lambda_{X}^{*}\right)+w\left(\lambda_{Y}^{*}\right)\right)(T-t)} \phi_{Z(T-t)}(u)}{K^{i u}(i u-\zeta-1)(i u-\zeta)} d u
\end{aligned}
$$

with $\zeta \in\left[-\frac{1}{\sigma_{Z}^{2}}\left(\beta_{Z}+\sqrt{\beta_{Z}^{2}+2 \sigma_{Z}^{2} \theta}\right),-1\right]$, under the condition that

$$
\frac{1}{\sigma_{Z}^{2}}\left(\beta_{Z}+\sqrt{\beta_{Z}^{2}+2 \sigma_{Z}^{2} \theta}\right)>1
$$

Using the same arguments, we can, analogous to (9), derive the European put option pricing formula for $\zeta \in\left[0, \frac{1}{\sigma_{Z}^{2}}\left(\sqrt{\beta_{Z}^{2}+2 \sigma_{Z}^{2} \theta}-\beta_{Z}\right)\right]$.

\section{Empirical Application}

For an empirical illustration, we consider a contract on the Nikkei 225 stock index quoted in U.S. dollars (USD) with the payoff settled in Japanese yen (JPY). ${ }^{2}$ Suppose $V(t)$ is the dollar-valued price process of the Nikkei 225 index and $F(t)$ the process of the JPY-USD exchange rate, where one JPY amounts to $F(t)$ dollar. We estimate market parameters using daily logreturns on the USD-valued Nikkei 225 index and the JPY-USD exchange rate from January 4, 2000 to June 21, 2013. USD-valued Nikkei 225 index prices are obtained by converting the original JPY-valued Nikkei 225 index levels into U.S. dollars using the JPN-USD exchange rate. The (scaled) price series are shown in Figure 1 and summary statistics of the daily log-returns are given in Table 1

\footnotetext{
${ }^{2}$ The options on the Nikkei 225 dollar futures traded on the Chicago Mercantile Exchange are an example of such a quanto product.
} 


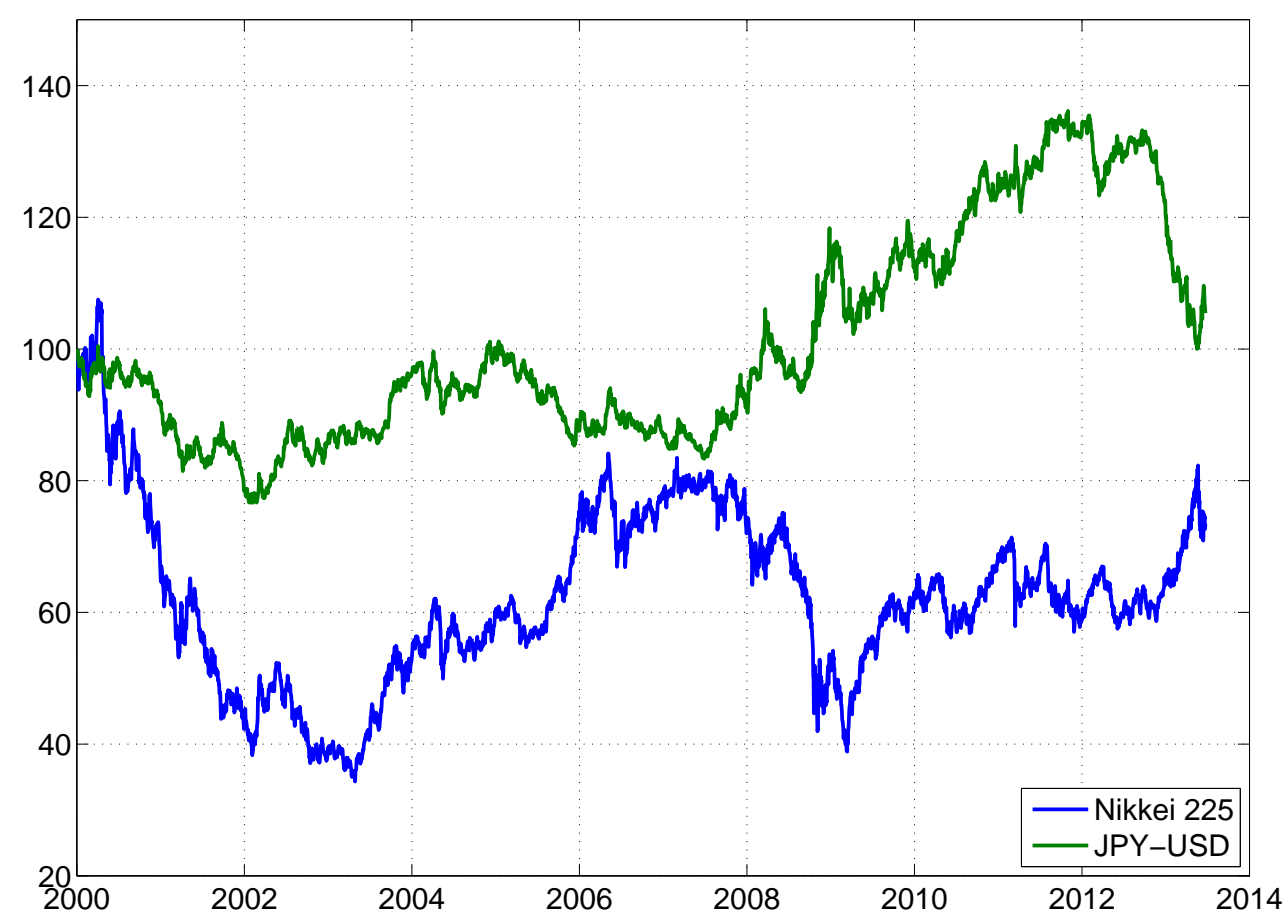

Figure 1: Price series (scaled) of the Nikkei 225 index and the JPY-USD exchange rate.

For the Black-Scholes model, we estimate $\mu_{X}, \mu_{Y}, \sigma_{X}, \sigma_{Y}$, and $\rho$ by the sample means, the sample standard deviations and the sample correlation. For the NTS model, the parameters $\mu_{X}, \mu_{Y}, \alpha, \theta, \beta_{X}, \beta_{Y}, \sigma_{X}$, and $\sigma_{Y}$ are estimated using maximum likelihood; and $\rho$ is calculated via Equation (2). The estimated Black-Scholes and NTS market parameters and their 95\%confidence intervals are reported in Table $2 .^{3}$

\footnotetext{
${ }^{3}$ Note that all estimates are expressed in annualized form. To obtain the $95 \%$ confidence intervals, we simulate 1,000 random, bivariate NTS vectors from the estimated NTS model via

$$
\left[\begin{array}{l}
X(\Delta t) \\
Y(\Delta t)
\end{array}\right]=\left[\begin{array}{l}
\mu_{X} \\
\mu_{Y}
\end{array}\right] \Delta t+\left[\begin{array}{l}
\beta_{X} \\
\beta_{Y}
\end{array}\right](\mathcal{T}(\Delta t)-\Delta t)+\left[\begin{array}{cc}
\sigma_{X} & 0 \\
0 & \sigma_{Y}
\end{array}\right]\left[\begin{array}{cc}
1 & 0 \\
\rho & \sqrt{1-\rho^{2}}
\end{array}\right]\left[\begin{array}{l}
\epsilon_{X} \\
\epsilon_{Y}
\end{array}\right] \mathcal{T}(\Delta t)
$$

where the subordinator $\mathcal{T}(\Delta t)$ is set to $\Delta t=1 / 250$ (i.e., the reciprocal of the number of trading days per annum), and $\epsilon_{X}$ and $\epsilon_{Y}$ are independent standard normal random variables. We repeat the simulation and the parameter estimation 1,000 times and obtain
} 
Table 1: Summary statistics for the daily log-returns on the Nikkei 225 index and the Japanese yen and U.S. dollar exchange rate over the period January 5, 2000 and June 21, 2013.

\begin{tabular}{lcc}
\hline \hline & Nikkei 225 & JPY-USD \\
\hline Mean & $-9.6686 \cdot 10^{-5}$ & $1.6645 \cdot 10^{-5}$ \\
Std. deviation & 0.0166 & 0.0068 \\
Skewness & -0.3912 & -0.0610 \\
Kurtosis & 7.9913 & 6.7307 \\
Minimum & -0.1371 & -0.0547 \\
$Q_{.01}$ & -0.0461 & -0.0185 \\
$Q_{.05}$ & -0.0263 & -0.0103 \\
$Q_{.1}$ & -0.0191 & -0.0078 \\
$Q_{.5}$ & $3.2992 \cdot 10^{-4}$ & $9.0306 \cdot 10^{-5}$ \\
$Q_{.9}$ & 0.0192 & 0.0078 \\
$Q .95$ & 0.0244 & 0.0105 \\
$Q_{.99}$ & 0.0395 & 0.0184 \\
Maximum & 0.1186 & 0.0360 \\
Correlation & \multicolumn{2}{c}{0.2851} \\
\hline
\end{tabular}

The $\alpha$-estimate of 1.4953 is well below 2 (the Gaussian case), indicating substantial fat-tailedness for the returns on the dollar-valued Nikkei index and the yen-dollar exchange rate. The negative $\beta_{N i}$-estimate of -0.3882 , with the 95\%-confidence interval excluding 0 , reflects the negative skewness of the Nikkei index returns, whereas the marginal JPN-USD distribution is practically symmetric. Hence, the dependence between the returns on the Nikkei and yen is not a simple linear one that can be fully described in terms of a correlation measure.

Graphs of the empirical bivariate density obtained via kernel-density estimation as well as the fitted Gaussian and NTS models are shown in Figure 2. It is evident that the Gaussian model cannot capture the pronounced peakedness of the empirical distribution. Having a fixed shape parameter,

1,000 sets of parameters. The 95\%-confidence intervals are, then, defined by the empirical $2.5 \%$ and $97.5 \%$ quantiles. The confidence intervals for the Gaussian estimates are obtained in an analogous manner. 
Table 2: Estimated market parameters and 95\% confidence intervals (in parentheses) for the Gaussian model and the normal tempered stable (NTS) models fitted to the Nikkei 225 index (subscript $N i$ ) and the JPY-USD exchange rate (subscript $F X$ ).

\begin{tabular}{ccc}
\hline \hline & Gaussian & NTS \\
\hline$\mu_{N i}$ & -0.0242 & -0.0231 \\
& $(-0.0290,-0.0190)$ & $(-0.1619,0.1194)$ \\
$\sigma_{N i}$ & 0.2630 & 0.2586 \\
& $(0.2594,0.2668)$ & $(0.2478,0.2673)$ \\
$\beta_{N i}$ & 0 & -0.3822 \\
& - & $(-0.7787,-0.0806)$ \\
$\mu_{F X}$ & 0.0042 & 0.0035 \\
& $(0.0022,0.0063)$ & $(-0.0570,0.0648)$ \\
$\sigma_{F X}$ & 0.1079 & 0.1065 \\
& $(0.1064,0.1095)$ & $(0.1018,0.1105)$ \\
$\beta_{F X}$ & 0 & 0.0494 \\
& - & $(-0.0911,0.2102)$ \\
$\rho$ & 0.2851 & 0.2971 \\
& $(0.2671,0.3031)$ & $(0.1748,0.3565)$ \\
$\alpha$ & 2 & 1.4953 \\
$\theta$ & - & $(1.0973,1.6517)$ \\
& - & 53.094 \\
& - &
\end{tabular}

the Gaussian model lacks flexibility and has to settle for a compromise when trying to fit the center and the tails of the distribution.

This is reflected in the goodness-of-fit properties of the fitted marginal distributions. We compute the Kolmogorov-Smirnov (KS) and the AndersonDarling $\left(\mathrm{AD}^{2}\right)$ test statistics, given by

$$
K S=\sum_{x}|\hat{F}(x)-F(x)|
$$

and

$$
A D^{2}=s \int_{-\infty}^{\infty} \frac{(\hat{F}(x)-F(x))^{2}}{F(x)(1-F(x))} d F(x),
$$

respectively, where $\hat{F}(x)$ is the empirical sample distribution, $F(x)$ the es- 
(a)

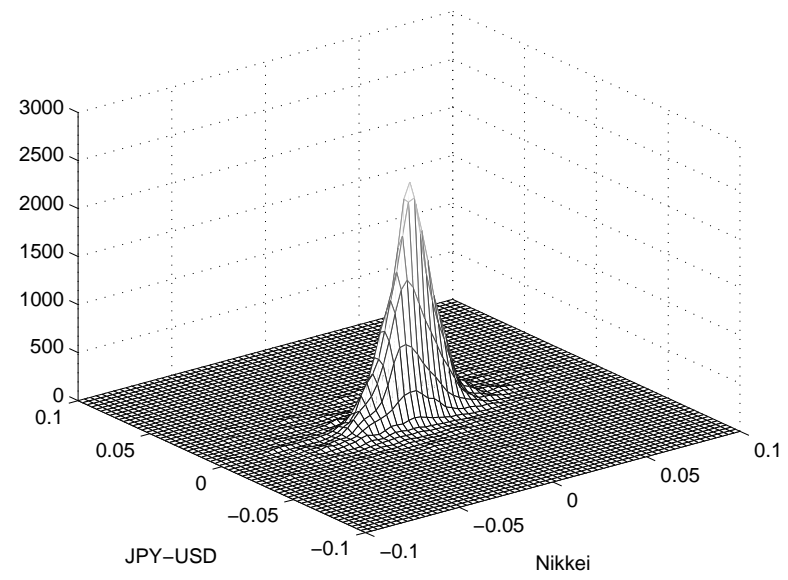

(b)

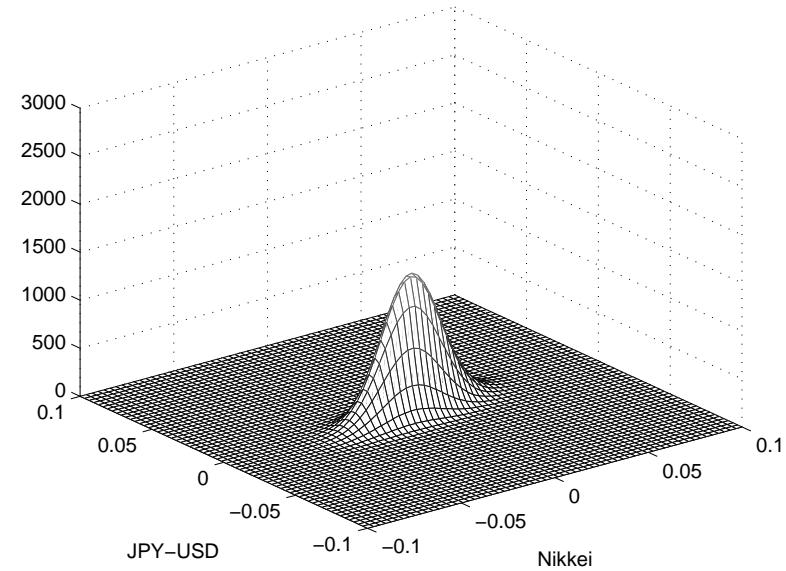

(c)

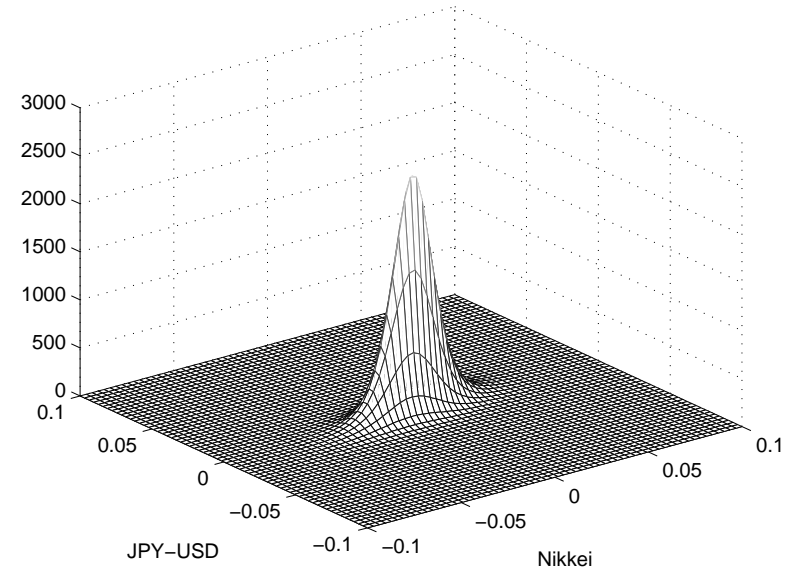

Figure 2: Fitted bivariate density function: (a) kernel density estimate, (b) Gaussian fit, and (c) NTS fit. 
Table 3: Goodness of fit measured by the Kolmogorov-Smirnov (KS) and Anderson-Darling $\left(\mathrm{AD}^{2}\right)$ statistics and their $p$-values (in parentheses) for the Gaussian model and the normal tempered stable (NTS) model fitted to the Nikkei 225 index and the JPY-USD exchange rate.

\begin{tabular}{lccc}
\hline \hline & & Gaussian & NTS \\
\hline Nikkei & KS & 0.0494 & 0.0118 \\
& & $(0.0000)$ & $(0.7638)$ \\
& $\mathrm{AD}^{2}$ & 16.37 & 0.3102 \\
& & $(0.0000)$ & $(0.9303)$ \\
JPY-USD & KS & 0.0499 & 0.0140 \\
& & $(0.0000)$ & $(0.5547)$ \\
& $\mathrm{AD}^{2}$ & 16.429 & 0.4410 \\
& & $(0.0000)$ & $(0.8073)$ \\
\hline
\end{tabular}

timated theoretical distribution, and $s$ the number of observations. ${ }^{4}$ The goodness-of-fit statistics for both models, together with their $p$-values, are also reported in Table 3. The $p$-values strongly indicate that the BlackScholes model is rejected by the data, whereas the NTS model is rather compatible with the data.

To derive risk-neutral parameters, we set the domestic (i.e., U.S.) riskfree rate to $r_{d}=0.25 \%$ and the foreign rate to $r_{f}=0.1 \%$. These were the respective central bank rates for the U.S. and Japan in June 2013. For the NTS model, we obtain $\lambda_{X}^{*}=-8.1181 \cdot 10^{3}$ and $\lambda_{Y}^{*}=-8.2020 \cdot 10^{3}$. These values satisfy Conditions RN.1 and RN.2. Setting $F_{f i x}=0.010214$, time-tomaturity to three months (i.e., $T-t=0.25$ ), the current underlying index price to $S(t)=13,230 \mathrm{JPY}$, and considering a range of in- and out-of-themoney strike prices, we derive European quanto call (and put) option prices for the Black-Scholes and the NTS model using (4) and (9).

The option prices implied by the two models are plotted in Figure 3;

\footnotetext{
${ }^{4}$ The calculation of the $p$-values for the $\mathrm{KS}$ and $\mathrm{AD}^{2}$ statistics is detailed in Marsaglia et al. (2003) and Marsaglia and Marsaglia (2004).
} 

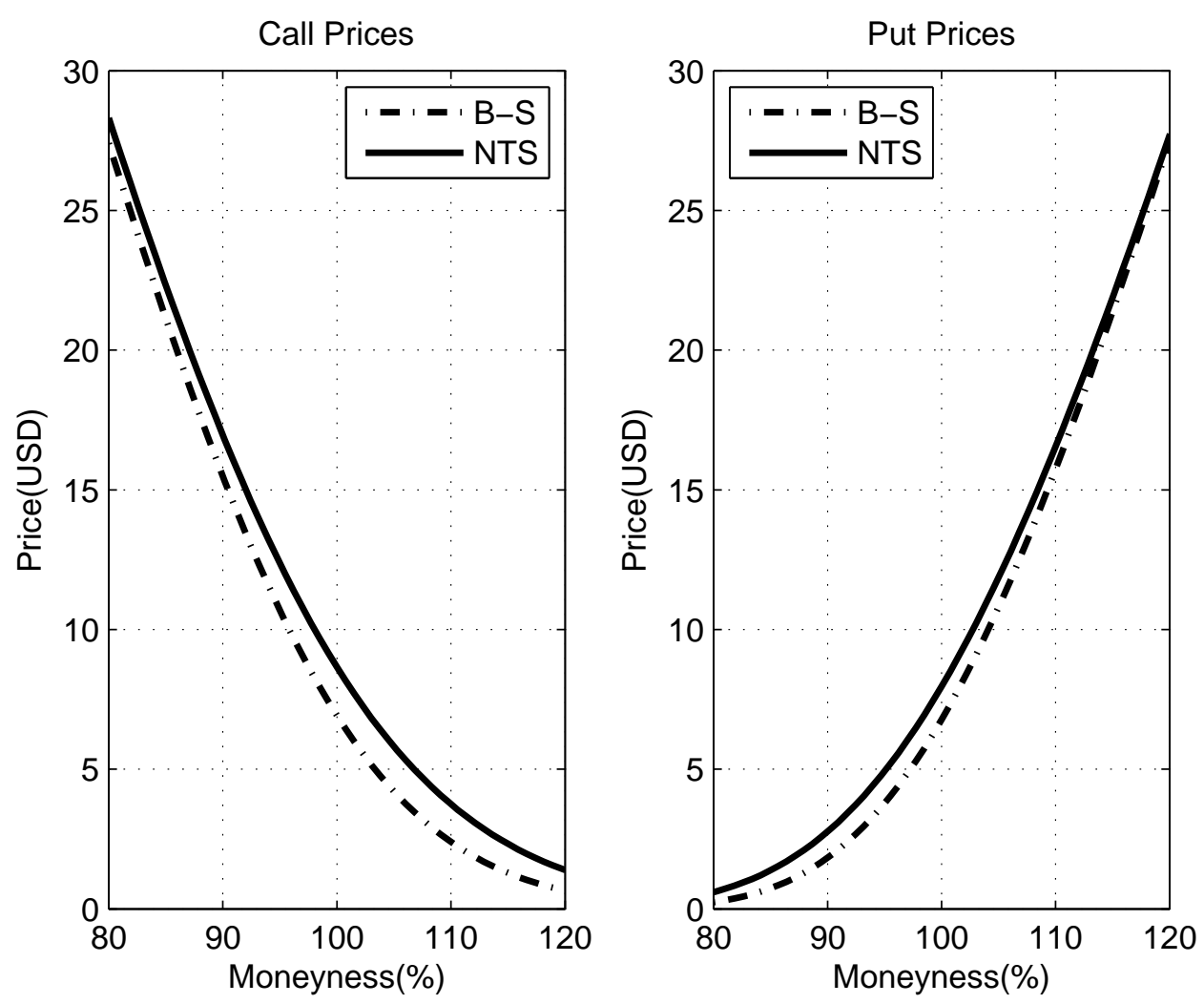

Figure 3: European quanto call prices (left) and European quanto put prices (right) implied by the Black-Scholes (B-S) model and the normal tempered stable (NTS) model as a function of moneyness, $K / S(0)$, where $K$ denotes the strike price and $S(0)$ the current underlying index level.

and differences (in percent) of the NTS prices relative to those from the Black-Scholse model are graphed in Figure 4. Both call and prices derived from the NTS model are higher than those of the Black-Scholes model. The differences increase the further we move out of money. This is due to the fact that the NTS model assigns higher probabilities to tail events. Figure 4 also demonstrates that the presence of asymmetries affects NTS option pricing. Relative to the BS-impled prices, out-of-the-money put options are valued higher than the corresponding out-of-the-money call options, which is a consequence of the Nikkei returns being negatively skewed. 


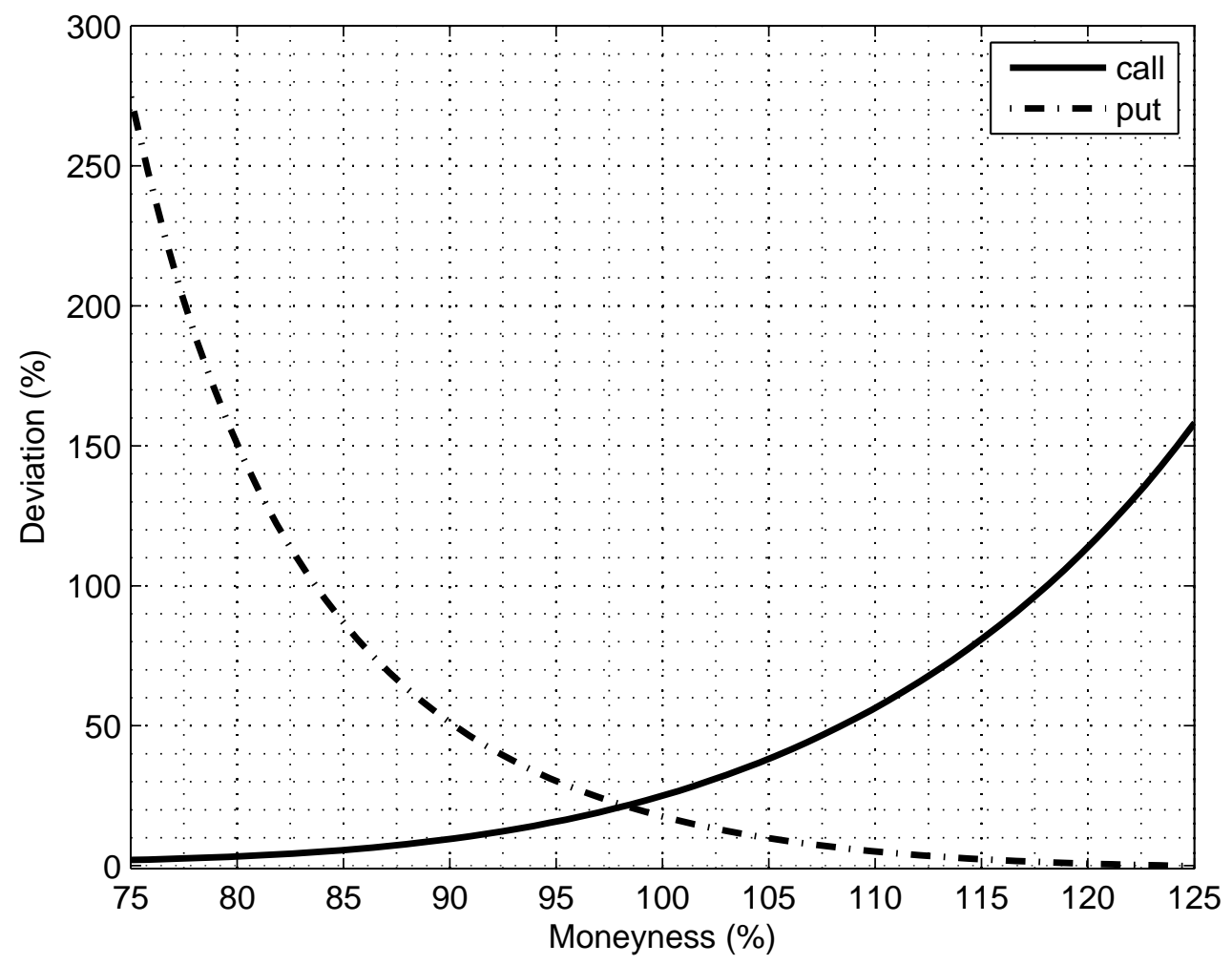

Figure 4: Relative deviations of European quanto call prices (solid line) and European quanto put prices (dash-dot) of the normal tempered stable model from the Black-Scholes prices as a function of moneyness.

Given the strong empirical support for the NTS model compared to the conventional Black-Scholes version, we suspect that NTS-based quantooption valuation is also more reliable - though, this will be subject of future research.

\section{Conclusion}

We have proposed an approach to pricing European quanto options based on the multivariate normal tempered stable (NTS) model. This allows us to capture both fat-tailedness and asymmetric dependence between the returns of the underlying asset and exchange rate. For an empirical illustration, we 
have estimated the market and the risk-neutral parameters for a quanto construction involving the Nikkei 225 index as underlying asset and the Japanese yen and U.S. dollar exchange rate. Whereas - based on two goodness-of-fit criteria - the Black-Scholes model is clearly rejected by the data, the NTS model could not be rejected at any reasonable confidence level. Quanto call and put prices derived for both the estimated NTS and the conventional Black-Scholes models turn out to differ substantially, with the NTS model giving rise to higher prices, as it pays more attention to the tail behavior of the instruments involved. Empirical analyses with observed quanto option transaction data and comparisons with alternative quanto pricing strategies are subject of future research.

\section{References}

Antonelli, F., Ramponi, A., Scarlatti, S., 2010. Exchange option pricing under stochastic volatility: a correlation expansion. Review of Derivatives Research 13 (1), 45-73.

Barndorff-Nielsen, O. E., Levendorskii, S., 2001. Feller processes of normal inverse Gaussian type. Quantitative Finance 1, 318 - 331.

Barndorff-Nielsen, O. E., Shephard, N., 2001. Normal modified stable processes. Economics Series Working Papers from University of Oxford, Department of Economics 72 .

Baxter, M., Rennie, A., 1996. Financial Calculus: An Introduction to Derivative Pricing. Cambridge University Press.

Black, F., Scholes, M., 1973. The pricing of options and corporate liabilities. The Journal of Political Economy 81 (3), 637-654. 
Carr, P., Madan, D., 1999. Option valuation using the fast fourier transform. Journal of Computational Finance 2 (4), 61-73.

Dimitroff, G., Szimayer, A., Wagner, A., 2009. Quanto option pricing in the parsimonious Heston model, Working Paper, Fraunhofer ITWM, Nr. 174. URL www.itwm.fraunhofer.de/fileadmin/ITWM-Media/Zentral/Pdf/ Berichte_ITWM/2009/bericht_174.pdf

Harmantzis, F., Miao, L., 2009. Dynamic asymmetric dependencies between equities and exchange rate markets.

URL http://ssrn.com/abstract=1339325

Hull, J., White, A., 1987. The pricing of options on assets with stochastic volatilities. Journal of Finance 42 (2), 281-300.

Kim, Y. S., Giacometti, R., Rachev, S. T., Fabozzi, F. J., Mignacca, D., 2012. Measuring financial risk and portfolio optimization with a non-Gaussian multivariate model. Annals of Operations Research 201 (1), 325-343.

Kim, Y. S., Rachev, S. T., Bianchi, M. L., Fabozzi, F. J., 2008. Financial market models with Lévy processes and time-varying volatility. Journal of Banking and Finance 32, 1363-1378.

Kim, Y. S., Volkmann, D., 2013. NTS copula and finance. Applied Mathematics Letters 26, 676-680.

Klebaner, F. C., 2005. Introduction to Stochastic Calculus with Applications, 2nd Edition. Imperial College Press.

Lewis, A. L., 2001. A simple option formula for general jump-diffusion and other exponential Lévy processes.

URL www.optioncity.net/pubs/ExpLevy.pdf 
Marsaglia, G., Marsaglia, J., 2004. Evaluating the Anderson-Darling distribution. Journal of Statistical Software 9 (2), 1-5.

Marsaglia, G., Tsang, W., Wang, G., 2003. Evaluating Kolmogorov's distribution. Journal of Statistical Software 8 (18).

Morales, L., 2007. The dynamic relationship between stock prices and exchange rates: evidence from four transition economies., paper presented to the Asociación Española de Economía y Finanzas (AEEFI), X Décimas Jornadas de Economia International, June 20-22, Madrid, Spain.

Park, J., Lee, Y., Lee, J., 2013. Pricing of quanto option under the hull and white stochastic volatility model. Communications of the Korean Mathematical Society 28 (3), 615-633.

Rachev, S. T., Mittnik, S., 2000. Stable Paretian Models in Finance. John Wiley \& Sons: New York. 